\title{
Instrument for high-speed recording of accommodation of the
} human eye

\section{Journal Article}

Author(s):

Monticone, Pier Paolo; Menozzi, Marino; Schlup, Martin

Publication date:

2010-04

Permanent link:

https://doi.org/10.3929/ethz-b-000018205

Rights / license:

In Copyright - Non-Commercial Use Permitted

Originally published in:

Biomedizinische Technik 55(2), https://doi.org/10.1515/BMT.2010.023 


\section{Instrument for high-speed recording of accommodation of the human eye}

\author{
Pier Paolo Monticone ${ }^{1, *}$, Marino Menozzi ${ }^{1}$ and \\ Martin Schlup ${ }^{2}$ \\ ${ }^{1}$ Technology and Innovation Management, ETH Zürich, \\ Zurich, Switzerland \\ ${ }^{2}$ Zentrum für Signalverarbeitung und Nachrichtentechnik, \\ Zürcher Fachhochschule für Angewandte Wissenschaft, \\ Winterthur, Switzerland
}

\begin{abstract}
We developed an instrument used for recording the dynamics of accommodation of the eye. With this new technique, a recording speed of up to $95 \mathrm{~Hz}$ can be achieved. As components used in our system are mechanically fixed, artifacts owing to movable mechanical parts are excluded. Recording samples demonstrate the potential use of this instrument. This instrument also enables assessment of gaze direction simultaneously with accommodation.
\end{abstract}

Keywords: accommodation dynamics; crystalline lens; gaze direction; infrared optometer; microfluctuations; vision.

\section{Introduction}

To produce a sharp retinal image, in human vision, the power of the eye is adjusted in accordance to the viewing distance. The process of adjustment of the power is termed accommodation. The mechanism of accommodation has intensively been investigated and modeled in the past $[2,3,8,11,14$, $16,24]$. Modeling of the accommodation in general and particularly modeling of the adequate stimulus of accommodation have been the focus of much research.

A basic question in modeling accommodation is the linearity of the accommodation plant. At first glance accommodation appears to be controlled by a low-pass filter with a delay, therefore showing linear behavior. However, Stark showed that, unlike in the case of a linear system, the transfer function varies with the amplitude of the stimulus, although the phase does not [27]. Stark ascribed this behavior to a memory-less (instantaneous) nonlinearity of the system and attempted a nonlinear servo analysis [21, 22, 28]. A substantial amount of data reported in the literature indicates that complex, nonlinear behavior could occur in many circumstances [7, 22, 26]. However, it soon became clear that the accommodation process as a whole is not as simple as a

\footnotetext{
*Corresponding author: Pier Paolo Monticone, TIM MTEC ETH Zürich, 8092 Zurich, Switzerland

Phone: +41-44-6322-531

E-mail: pmonticone@ethz.ch
}

linear low-pass filter. Moreover, there are adaptive learning effects, as revealed by Stark in 1968 [27], as the subject can anticipate stimulus changes, which is the case when the stimulus varies periodically in time. In this case, there is a reduction of the phase lag and this could also enhance the gain [11]. A sophisticated model of the accommodation was presented by Schor et al. in 1992 [25]. The model describes the accommodation as driven by two complementary processes: spatiotopic (body referenced) stimulus of proximal perception and a retinotopic (retina referenced) stimulus of blur and disparity. Spatiotopic stimuli initiate changes in viewing distance (coarse, primary control), whereas retinotopic stimuli are used for correcting residual fixation errors (fine, secondary control).

Information about the neurophysiology of the accommodation can be obtained by studying the dynamic characteristics of accommodation. The relationship between the peak velocity and the response amplitude, the so-called main sequence, is of particular interest in understanding how physiological responses are generated [15]. The relationship is also used to distinguish between normal and abnormal responses [4]. In accordance to Nyquist's theorem [6, 20], an accurate estimation of the dynamics of accommodation requires a minimum sampling rate.

Sampling rates of instruments used to record dynamics of accommodation, the so-called optometers, vary based on the techniques used to detect the accommodation $[9,10,12]$. For instance, the first generation of the commercially available photorefractor (PowerRefractor, Multichannel Systems, Reutlingen, Germany) is based on the evaluation of the distribution of light within the video image of the pupil and has a sampling rate of $25 \mathrm{~Hz}$ [11]. Recently, wave-front aberrometers have been launched on the market by means of which a dynamic estimation of accommodation is possible at a rate of $11 \mathrm{~Hz}$ [13].

Sampling rate is of crucial importance when using recorded data in modeling accommodation. Suryakumar et al. recorded the step response of the accommodation with a custom designed high-speed photorefractor at $75 \mathrm{~Hz}$ [23]. They subsampled recorded signals at $25 \mathrm{~Hz}$ to compare the effects of a lower sampling rate on the temporal characteristics of the signal. Suryakumar et al. concluded that faster sampling improves the accuracy of dynamic properties of the accommodation and that the peak velocity and acceleration are underestimated by $24 \%$ and $35 \%$, respectively, when subsampling at $25 \mathrm{~Hz}$. Furthermore, subsampling causes a time shift of the acceleration peak.

With the exception of ultrasonic devices used in biometry of the eye [29], objective and automatic assessment of accommodation is based on an analysis of light refracted by the dioptric media of the eye. In particular, light, mostly 
infrared, is projected into the eye causing reflections which are then analyzed by an optometer to determine accommodation. To prevent damage, energy of the light penetrating the eye is limited. Owing to low reflectivity of the retina, high sensitivity detectors are required in optometers. Given cited limitations, large detectors which are continuously adjusted in position are used in optometers to maximally exploit reflected light. Control of the position of the detector is a possible cause for artifacts in measurement.

In this paper, we present a custom made high-speed optometer allowing the recording of accommodation up to $95 \mathrm{~Hz}$. Our optometer has no moving parts, therefore excluding artifacts owing to mechanical properties such as resonance or inertia. While tracking the accommodation, our optometer allows a simultaneous detection of the shape and the position of the pupil and therefore also an estimation of gaze direction and pupillary diameter.

\section{Materials and methods}

\section{Principle and components}

The principle consists of analyzing an image produced by an object, which is placed at a fixed distance from an image sensor and which is illuminated by light exiting the pupil of the eye under test. The size of the image varies with the vergence of the light exiting the pupil and therefore varies with the accommodation.

The three main components in our device are a source of light, a plane mirror with a cylindrical hole (pinhole) and an optical sensor.

The laser diode QL78D6SA (Roithner LaserTechnik $\mathrm{GmbH}$, Vienna, Austria) is used as light source. The laser emits a power of $5 \mathrm{~mW}$ at a wavelength of $780 \mathrm{~nm}$. Laser power is adjusted to radiate $450 \mu \mathrm{W}$ at the cornea, which is below the maximum permissible power according to ANSI standard Z136.1 [1]. Before each measurement the laser power is measured using the LaseCheck power meter from Coherent Inc. (Roithner LaserTechnik GmbH). The mirror is a polished metal layer. A hole in 1-mm diameter is drilled at an angle of $45^{\circ}$ while the mirror is squeezed between two metal prisms. A Sony ICX204 AL/AK CCD (camera scA1000-30gm/gc, Basler AG, Ahrensburg, Germany) acts as an optical sensor. The arrangement of the three components is represented schematically in Figure 1. The laser is placed behind the mirror. The laser beam passes the hole in the mirror and penetrates the eye. To eliminate disturbing reflections the eye is adjusted in such a way that the laser beam hits the cornea slightly below the optical axis. After having passed through the various parts of the inner eye the laser beam hits the retina and is then partially reflected back. The reflected light is diffuse and exits the eye forming a cone with the pupil as base. The inclination of the surface of the cone is controlled by the optical power of lens and cornea. The exiting beam is then reflected by the pinhole mirror and projected on the image sensor. The hole in the mirror leaves a part of the reflected beam dark, therefore imaging a dark spot in the image of the pupil appearing on

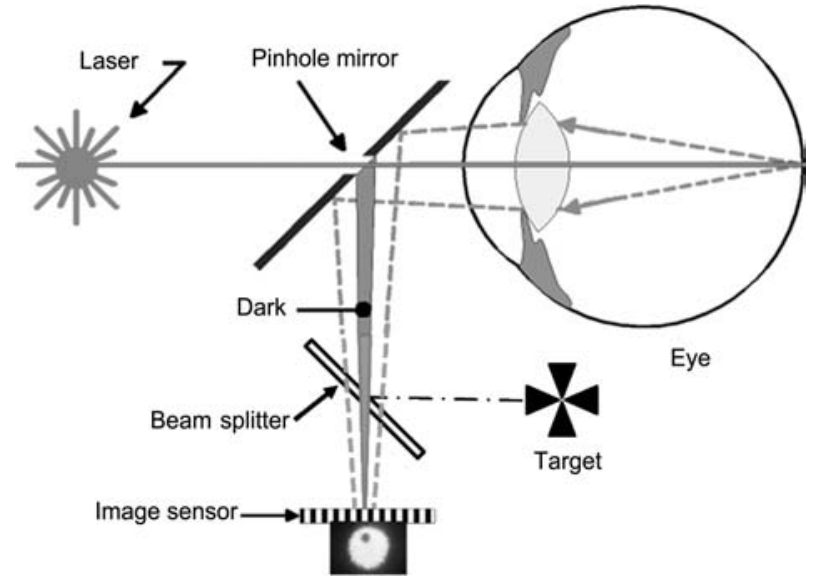

Figure 1 Schematic diagram of the device used to record accommodation consisting of a laser light source, a pinhole mirror, and an image sensor (CCD camera).

Fixation targets are presented at various distances using a beam splitter. The photograph at the bottom of the scheme provides an example of a recorded image.

the sensor. The size of the dark spot varies with the power of the eye. Sensor data are transmitted using a Gigabit Ethernet $(1 \mathrm{~Gb} / \mathrm{s})$ to a computer that records the video for off-line processing.

The three components are fixed on a bench. The bench includes a bite bar allowing a stable position of the eye with regard to the recording instrument. Centering of the laser beam and of the camera is achieved by means of an $x-y$ table. To stimulate the accommodation, a beam splitter $(\mathrm{T}=95 \%)$ is introduced between the pinhole mirror and the image sensor enabling presentation of fixation targets at different viewing distances.

\section{Image processing}

As mentioned above, the camera provides a video of the image of the pupil including the dark spot resulting from the hole in the mirror. The gray level video is recorded using a PC and is analyzed off-line frame by frame. The analysis consists of detecting the border of the image of the pupil and the border of the dark spot. Detection is performed using image processing software. We used the function "Circular Edge (Spoke)" implemented in LabView 8.5 (National Instruments Corp., Austin, TX, USA) fitting the border of the pupil and the border of the dark spot with a circle. In the function "Circular Edge (Spoke)", a center of a circle is assumed and radii are plotted at a given number of equally distributed meridians. The edge of the image (hole or pupil) is detected along each meridian. Detected edges are used to least square fit of a circle. Owing to noise resulting from the speckle pattern and other optical factors, parameters used by the function "Circular Edge (Spoke)", such as number of meridians used or steepness of the brightness transition, require an appropriate setting procedure. To optimize these parameters we followed two steps: a manual step and an automatic step. 
In the manual step the parameters are chosen to achieve best fit by eye of the circle with the edge of the image of the hole. The set of results found in the first step are used as initial values in the second step. Purpose-written software was used in the second step aiming to optimize the abovementioned parameters to achieve an optimum fit of the circle. The goodness-of-fit is defined as the sum of variances in the sequence of analyzed frames, resulting from the differences of the radius of the fitted circle and the distance of detected edges from the center of the circle. To keep the elaboration cost at a reasonable level, the software does not check for found minimum to be an absolute minimum. Furthermore, only two key parameters are optimized: "edge strength" and "steepness".

\section{Practical limitation}

In the realization of the system we faced some limitations. A first constraint is that tracking the position of the eye requires the pupil to be fully imaged on the sensor. Therefore, the image of the pupil must be centered on the CCD and the size of the image must be smaller than the size of the CCD. It is possible to minify the image of the pupil by increasing the distance of the eye with regard to the CCD or by introducing a lens. Alternatively the CCD can be replaced by a CCD of larger size.

Another constraint is the space in-between the eye and the CCD. Approaching the CCD to the eye improves resolution in assessing accommodation. However, the closest distance of placement of the CCD should consider the closest point the eye is able to focus, to prevent the image of the pupil to degenerate to a point on the CCD.

A further limitation arises when using the instrument at maximum recording speed. In the set-up used for this study the CCD is at $90 \mathrm{~mm}$ from the cornea enabling recording of the accommodation from 1 to 4 diopters (D). To achieve a sampling rate of $95 \mathrm{~Hz}$, a subsample of the total area of the CCD is selected, which contains the image of the hole. In this condition, the position of the eye cannot be tracked because the selected area does not include the edge of the pupil.

\section{Functional tests}

Functional tests of the apparatus are carried out in view of the future application of the instrument, which comprises investigations of the accommodation process and its crosslink to gaze direction. Velocity and acceleration of the accommodation response are parameters which are relevant to investigation of accommodation dynamics. Further information about the accommodation process is gained by temporal analysis of accommodation behavior during the steady-state fixation of a target with constant viewing distance.

Functional tests are carried out using the eye of a young subject (27 years old). The test was performed in accordance with the Declaration of Helsinki ethical guidelines (World Medical Association Declaration of Helsinki regarding ethical conduct of research involving human subjects and/or ani- mals) and was approved by the local ethics committee (ETH Zurich). After explanation of the nature and possible consequences of the experiment, informed consent was obtained from the subject. All measurements were carried out under monocular viewing conditions using the subject's right eye and within the range of accommodation of the subject. To estimate the range of accommodation, the subject's amplitude of accommodation was measured using a push-up ruler and the refractive error of the right eye was assessed using the automatic refraction unit Nidek AR-1000 (von Hoff Augenoptik International, Schlieren, Switzerland). Considering the subject's amplitude of accommodation ( $>5$ D) and the refractive error (-0.75 D spherical equivalent), selected stimulus range varied between $1 \mathrm{D}$ and $4 \mathrm{D}$.

The subject was fixed using a bite bar to the board of the instrument. By means of the $x-y$ table the pupil of the eye is centered on the image sensor of the camera. A first experiment was assigned to record the static response of accommodation as well as to estimate stability of gaze direction during steady-state fixation of a target presented at a fix viewing distance. Additionally, the first experiment was used for calibration of the instrument. A beam splitter was introduced between the camera and the pinhole mirror enabling the presentation of a Maltese cross at either $1 \mathrm{~m}, 50 \mathrm{~cm}, 33 \mathrm{~cm}$, or $25 \mathrm{~cm}$. The four viewing distances stimulate accommodation at $1 \mathrm{D}, 2 \mathrm{D}, 3 \mathrm{D}$, and $4 \mathrm{D}$, respectively. For each viewing distance, the camera recorded four samples of 800 images using a sample frequency of $95 \mathrm{~Hz}$. Owing to band width limitation, the CCD camera used binning mode $(2 \times 2$ aggregation of pixels) and was programmed to process only the part of the image including the image of the pinhole. Before starting the experiment, a full frame picture was recorded and used to check the proper setting.

In a second experiment, step response of accommodation was recorded by presenting two Maltese crosses in two different viewing distances. The centers of the two Maltese crosses were aligned with the line of sight of the subject's eye. The first Maltese cross was placed at a viewing distance of $1 \mathrm{~m}$, therefore requiring an accommodation of $1 \mathrm{D}$. The second Maltese cross was placed at $25 \mathrm{~cm}$, therefore requiring an accommodation of $4 \mathrm{D}$. Illumination of Maltese crosses was controlled by an electronic switch, so that only one of the two Maltese crosses was visible at one time. The electronic switch was triggered by the digital output signal "exposure active"' of the camera. During measurements, the target was switched from $1 \mathrm{D}$ to $4 \mathrm{D}$. After $4 \mathrm{~s}$ the target was switched back to $1 \mathrm{D}$ again. During the experiment, the camera was programmed as reported in the first experiment and therefore sampled images at a frequency of $95 \mathrm{~Hz}$.

\section{Results}

Results of the first experiment, in which the static response of the accommodation was assessed, are shown in Figure 2. The four photographs in Figure 2 show samples of images taken before starting the experiment. The dark spot within the pupil decreases in diameter as accommodation increases. 


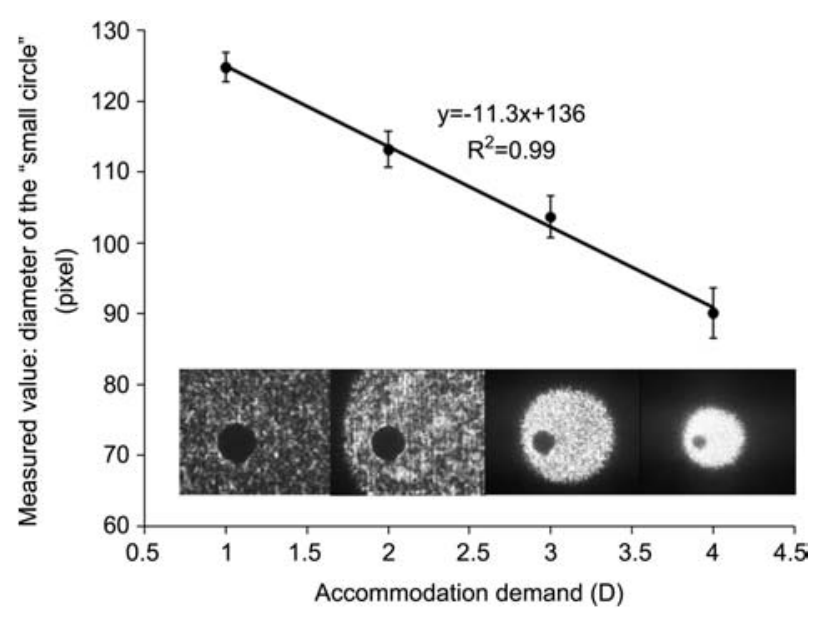

Figure 2 Results of the first experiment, in which static response of the accommodation was studied.

The graph plots the average diameter of the circular dark spot within the pupil in pixels as a function of the demand of accommodation. The four pictures show a sample of the whole picture taken before starting the experiment while fixating the target in each of the four viewing distances. The error bars denote the standard deviations $( \pm)$.

This effect is as a result of an increasing vergence of the beam exiting the pupil. Variation of the pupillary diameter with viewing distance is due, in part, to the phenomenon of pupillary constriction appearing when focusing near objects and, in part, to the increased vergence of the light exiting the pupil. The graph in Figure 2 plots the diameter of the black spot within the bright pupil as function of the accommodation demand resulting from given viewing distance. Each data point in Figure 2 is the average of 3200 measurements. Data points are reliably $\left(r^{2}=0.99\right)$ fitted by means of a line. The slope of the line is 11.3 pixels per accommodation power in diopters.

From results of the first experiment we learn that accommodation fluctuates by $0.25 \mathrm{D}$ (standard deviation) around the average value. As these fluctuations are a result of noise and fluctuations of accommodation are a result of difficulty in keeping accommodation fix, we can conclude that the accuracy of the instrument is better than $0.25 \mathrm{D}$.

As can be seen in Figure 2, there is a variation of the standard deviation of accommodation with demand of accommodation. This effect is, in part, due to the variation in image size with demand of accommodation and to the constant size of the CCD pixel. Also, the effect of speckle becomes more important in a small image compared with a large image. Therefore, constant pixel size and speckle reduce the accuracy of the instrument at high values of accommodation. However, we believe that the increased standard deviation of the measurements is mainly a result of the microfluctuations of the accommodation, which increase in amplitude with increasing accommodation.

Measurements recorded while the eye was steady-state fixating the target presented at a viewing distance of $25 \mathrm{~cm}$ were used for spectral analysis. For this purpose, spectra of four measurements, each lasting approximately $8 \mathrm{~s}$ and sampled at a rate of $95 \mathrm{~Hz}$, were computed after removing the linear trend and applying a Hann window. The result of the spectral analysis is shown in Figure 3. Plotted spectrum was normalized accounting for the number of recorded samples. According to the literature [6], bands below $1 \mathrm{~Hz}$ and at approximately $1.5 \mathrm{~Hz}$ are probably a result of small fluctuations of the power of the eye, the so-called microfluctuations of the accommodation.

From the same records used for the spectral analysis of steady-state accommodation, the center of the pupil was detected and converted in gaze direction using Gullstrand's simplified eye model reported by Methling [18]. Gaze direction was plotted as function of time in Figure 4. Data were low-pass filtered using a cut-off value of $9.5 \mathrm{~Hz}$. Standard deviation of the movement was $6.3 \mathrm{mrad}$.

Results of the second experiment, in which the dynamic response of accommodation was studied, are depicted in Figure 5. The data in Figure 5 are the average of 25 runs. The effect of averaging the measurement brings an accuracy of $0.25 \mathrm{D} /(25)^{1 / 2}=-0.05 \mathrm{D}$. At time $\mathrm{t}=0 \mathrm{~s}$ the distance of the target is switched from $1 \mathrm{~m}(1 \mathrm{D})$ to $25 \mathrm{~cm}(4 \mathrm{D})$. After $4 \mathrm{~s}$ the distance is switched back to $1 \mathrm{~m}$. An approximate estimate by eye of reaction time reveals a response of the accommodation of around $250 \mathrm{~ms}$. The rise and fall times, between $10 \%$ and $90 \%$ of maximum of amplitude, are around $200 \mathrm{~ms}$.

The time derivative of the accommodation response, which is the velocity of accommodation, is plotted in Figure 6. Because derivation enhances noise in particular in the high frequency domain, data presented in Figure 6 are filtered by using a moving average filter with a window of $0.1 \mathrm{~s}$. From data plotted in Figure 6, we can estimate that the absolute value of the maximum velocity of accommodation is approximately $13 \mathrm{D} / \mathrm{s}$ and appears around $0.4 \mathrm{~s}$ after altering the viewing distance of the target.

Acceleration of accommodation is computed by means of the second time derivative of the accommodation response reported in Figure 5. Again, results need filtering because the derivative enhances noise. In the case of the second derivative, noise amplification is proportional to the square of the frequency. However, as can be seen in Figure 7, acceleration data appear noisy even after filtering with a $0.2 \mathrm{~s}$

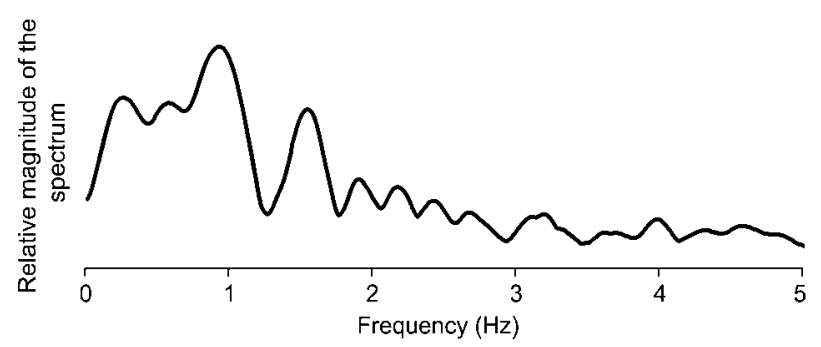

Figure 3 Spectrum of steady-state accommodation when fixating a target presented at a viewing distance of $25 \mathrm{~cm}(4 \mathrm{D})$.

Data represent the average of the spectra of four measurements, in which accommodation was recorded during $8 \mathrm{~s}$ using a sampling rate of $95 \mathrm{~Hz}$. 


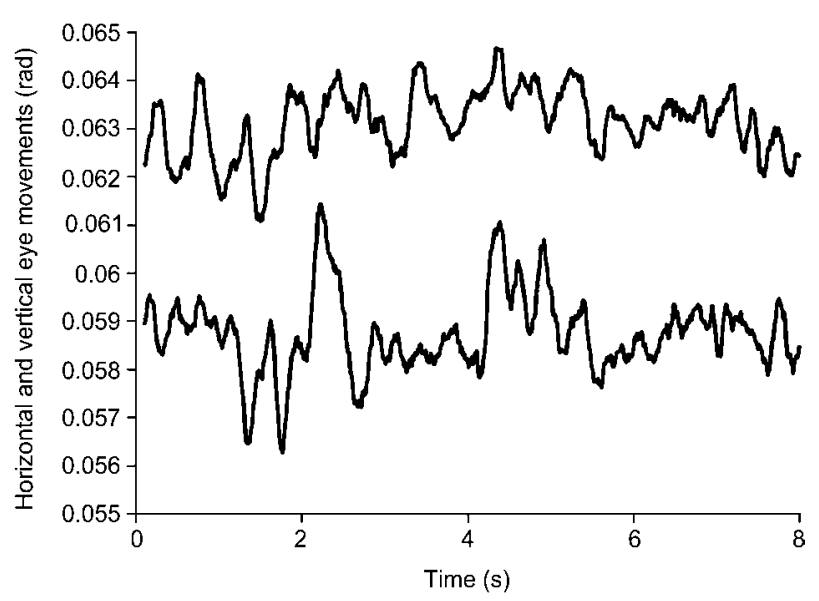

Figure 4 Gaze direction as function of time recorded during steady-state fixation of a target presented at a viewing distance of $25 \mathrm{~cm}$.

Data are low-pass filtered using a cut-off value at $9.5 \mathrm{~Hz}$ (moving average window of 10 points). The standard deviation of the movements is approximately $6.3 \mathrm{mrad}$.

moving average window. It is therefore difficult to give an accurate estimate for the acceleration of accommodation. Probably the absolute value of the maximum acceleration is within the range of 40 to $80 \mathrm{D} / \mathrm{s}^{2}$.

\section{Discussion}

In this paper, we presented a new optometer used to record the accommodation of the eye. The optometer was calibrated using a human eye. In the first experiment, accommodation was recorded during steady-state fixation at four different viewing distances. In the second experiment, accommodation was recorded during altering of the viewing distance between $1 \mathrm{~m}$ and $4 \mathrm{~m}$.

Fit of data of the first experiment demonstrates linearity of the method with regard to the power of accommodation in diopters. Slope of fit is approximately 11.3 pixels/D denoting a resolution of better than 1 pixel per $0.1 \mathrm{D}$. Owing to used image processing methods, we are confident that

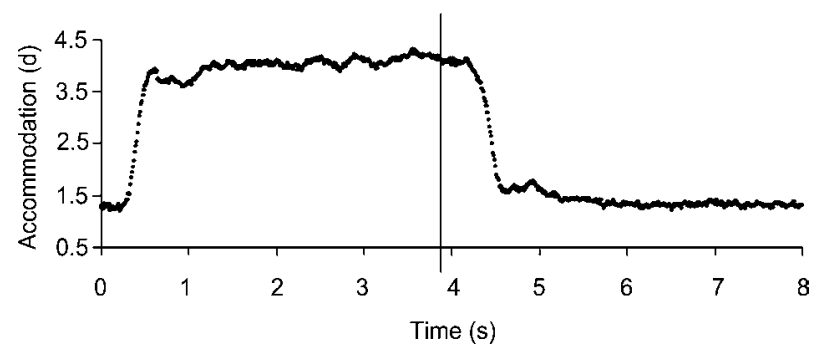

Figure 5 Results of the second experiment, in which dynamic accommodation response was studied by altering the target distance from $1 \mathrm{~m}(1 \mathrm{D})$ to $25 \mathrm{~cm}(4 \mathrm{D})$ and back to $1 \mathrm{~m}$ at $\mathrm{t}=0 \mathrm{~s}$ and $\mathrm{t}=4 \mathrm{~s}$, respectively.

The average of 25 measurements of accommodation is plotted as function of time.

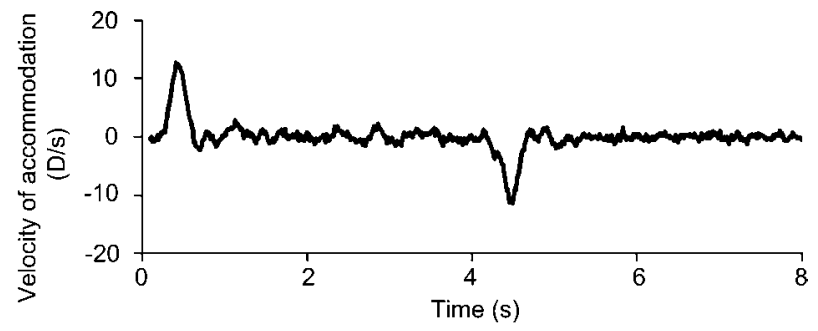

Figure 6 Velocity of the accommodation computed from the signal reported in Figure 5 and filtering the velocity using a cumulative moving average with a window length of $0.1 \mathrm{~s}$.

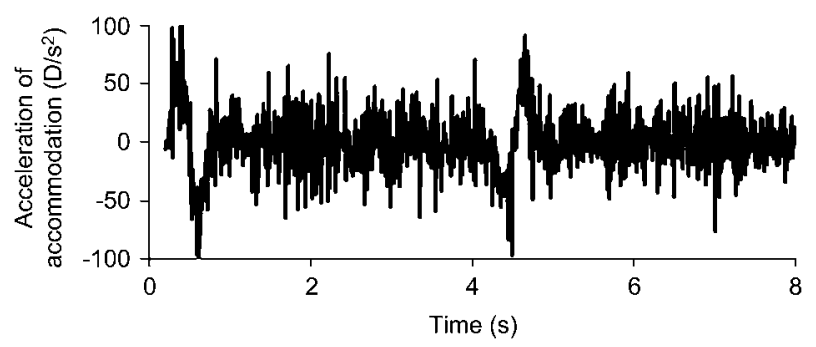

Figure 7 Acceleration of the accommodation computed from data reported in Figure 5 after filtering results using a cumulative moving average with a window length of $0.2 \mathrm{~s}$.

resolution is much better than $0.1 \mathrm{D}$. Recorded accommodation during steady-state fixation has been shown to fluctuate with an amplitude of approximately $0.25 \mathrm{D}$. If so-called microfluctuations do exist, amplitude of microfluctuations is probably less than $0.25 \mathrm{D}$. Spectrum of accommodation recorded during steady-state fixation demonstrates typical peaks of microfluctuations [23] at approximately $0.9 \mathrm{~Hz}$ and $1.5 \mathrm{~Hz}$. Dynamic properties of accommodation, such as latency and velocity, are in accordance to experimental results reported in the literature $[5,7,26]$ or to values used in modeling accommodation [24].

Using measurements of the first experiment, we assessed horizontal and vertical displacements of the pupil to cause a shift in gaze direction of approximately $6.3 \mathrm{mrad}$ (standard deviation of mean), which compares well to the spontaneous saccades during steady-state fixations reported in the literature $[17,19]$.

The presented instrument has proven functionality and results of recorded data compare well with the literature. Owing to the principle of measurement, instrumental artifacts in dynamic properties of accommodation are excluded. Owing to spherical aberration of the eye, artifacts could appear when the eye moves during measurement. However, as the position of the pupil and accommodation are recorded simultaneously, a compensation for this artifact is possible and will be done in future research. Further developments concern evaluation of techniques to compute velocity and acceleration using recorded signals.

\section{Acknowledgments}

This study was supported by grants from the Swiss National Science Foundation (SNSF) No. 320030-116423. 


\section{References}

[1] American National Standard for Safe Use of Lasers. ANSI Z136.1. 2007.

[2] Arnulf A, Dupuy O, Flamant F. Les microfluctuations d'accommodation de l'oeil et l'acuité visuel pour les diamétres pupillaires naturelles. C R Hebd Seanc Acad Sci 1951; 232: 339-341.

[3] Arnulf A, Santamaria J, Bescos J. A cinematographic method for the dynamic study of the image formation by the humaneye - microfluctuation of accommodation. J Optics - Nouv Rev Optique 1981; 12: 123-128.

[4] Bahill AT, Clark MR, Stark L. The main sequence, a tool for studying human eye movements. Math Biosci 1975; 24: 191204.

[5] Bharadwaj SR, Schor CM. Acceleration characteristics of human ocular accommodation. Vision Res 2005; 45: 17-28.

[6] Campbell FW, Robson JG, Westheimer G. Fluctuations of accommodation under steady viewing conditions. J Physiol (Lond) 1959; 145: 579-594.

[7] Campbell FW, Westheimer G. Dynamics of the accommodation responses of the human eye. J Physiol (Lond) 1960; 151: 285-295.

[8] Charman WN, Radhakrishnan H. Fluctuation in accommodation: a review. Ophthal Physiol Opt 1988; 9: 153-164.

[9] Choi M, Weiss S, Schaeffel F, Seidemann A, Howland HC. Laboratory, clinical, and kindergarten test of a new eccentric infrared photorefractor (PowerRefractor). Optom Vis Sci 2000; 77: 537-548.

[10] Collins G. The electronic refractometer. Br J Physiol Opt 1937; 1: 30-40.

[11] Franzen O, Richter H, Stark L. Accommodation and vergence mechanisms in the visual systems. 1st edition. Basel: Birkhäuser 2000.

[12] Gekeler F, Schaeffel F, Howland HC, Wattam Bell J. Measurement of astigmatism by automated infrared photoretinoscopy. Optom Vis Sci 1997; 74: 472-482.

[13] Iskander DR, Collins MJ, Morelande MR, Zhu M. Analyzing the dynamic wavefront aberrations in the human eye. IEEE Trans Biomed Eng 2004; 51: 1969-1980.

[14] Iwasaki T, Kurimoto S. Objective evaluation of eye strain using measurements of accommodative oscillation. Ergonomics 1987; 30: 581-587.

[15] Kasthurirangan S, Vilupuru AS, Glasser A. Amplitude dependent accommodative dynamics in humans. Vis Res 2003; 43: 2945-2956.

[16] Kotulak JC, Schor CM. Temporal variations in accommodation during steady-state conditions. J Opt Soc Am 1986; 3: 223-227.

[17] Menozzi M, Rhyner K, Joestl A, Stähli H, Bergande E. A simple video based system for examining irregularities in very slow, smooth pursuit eye movements in cancer patient. Biomed Tech 2008; 53: 1-7.

[18] Methling D. Bestimmen von Sehhilfen. 2nd edition. Stuttgart: Enke Verlag 1996.

[19] Moeller F, Laursen M, Tygesen J, Sjolie A. Binocular quantification and characterization of microsaccades. Graefes Arch Clin Exp Ophthalmol 2002; 240: 765-770.

[20] Nyquist H. Certain topics in telegraph transmission theory. Trans Am Inst Electr Eng 1928; 47: 617-644.

[21] O'Neill WD, Brodkey JS. Nonlinear servo analysis of the mechanics of accommodation. Vis Res 1970; 12: 307-318.

[22] O'Neill WD, Sanathanan CK, Brodkey JS. A minimum variance, time optimal, control system mode of human lens accommodation. IEEE Trans Syst Sci Cybern 1969; 5: 290-299.

[23] Suryakumar R, Meyers JP, Irving EL, Bobier WR. Application of video based technology for the simultaneous measurement of accommodation and vergence. Vis Res 2007; 47: 260-268.

[24] Schor CM, Bharadwaj SR. Pulse step models of control strategies for dynamic ocular accommodation and disaccommodation. Vis Res 2006; 46: 242-258.

[25] Schor CM, Cormack LK, Stevenson SB. Negative feedback control model of proximal convergence and accommodation. Ophthal Physiol Opt 1992; 12: 307-318.

[26] Shirachi D, Liu J, Lee M. Accommodation dynamics. 1. Range nonlinearity. Am J Optom Physiol Opt 1978; 55: 631-641.

[27] Stark L. Neurological control systems: studies in bioengineering. New York: Plenum Press 1968: 185-230.

[28] Stark L, Takahashi Y, Zames G. Nonlinear servoanalysis of human lens accommodation. IEEE Trans Syst Sci Cybern 1965; $1: 75-83$.

[29] van der Heijde GL, Beers APA, Dubbleman M. Microfluctuations of steady state accommodation measured with ultrasonography. Ophthalmic Physiol Opt 1996; 16: 216-221.

Received September 18, 2009; accepted December 21, 2009 